\title{
ON $H$-CLOSED SPACES
}

\author{
JAMES E. JOSEPH
}

\begin{abstract}
A characterization of $H$-closed spaces in terms of projections is given along with relating properties.
\end{abstract}

Introduction. The primary purpose of this paper is to give a characterization of $H$-closed spaces which is an analogue to the following theorem for compact spaces: A space $X$ is compact if and only if the projection from $X \times Y$ onto $Y$ is a closed function for every space $Y[9, \mathrm{p} .21]$.

Following the notation of [6], we utilize the notion of $\theta$-closed subsets of a topological space from [11, p. 103] and our characterization is stated as follows:

TheOREM. A Hausdorff space $X$ is $H$-closed if and only if for every space $Y$, the projection from $X \times Y$ onto $Y$ takes $\theta$-closed subsets onto $\theta$-closed subsets.

Throughout, $\operatorname{cl}(K)$ will denote the closure of a set $K$.

\section{Preliminary definitions and theorems.}

Definition 1. A net in a topological space is said to $\theta$-converge $(\theta$ accumulate) [6, Definition 3] to a point $x$ in the space if the net is eventually (frequently) in $\operatorname{cl}(V)$ for each $V$ open about $x$.

Definition 2. A point $x$ in a topological space $X$ is in the $\theta$-closure $[11, \mathrm{p}$. 103] of a set $K \subset X(\theta-\operatorname{cl}(K))$ if $\operatorname{cl}(V) \cap K \neq \varnothing$ for any $V$ open about $x$.

Definition 3. A subset $K$ of a topological space is $\theta$-closed [11, p. 103] if it contains its $\theta$-closure (i.e., $\theta-\operatorname{cl}(K) \subset K$ ).

The following theorems give some parallels of properties of closure and closed sets in a topological space for $\theta$-closure and $\theta$-closed sets in the space and some relationships between these notions. The proofs of these theorems are straightforward and are omitted [11, Lemmas 1, 2, 3].

THEOREM 1. A point $x$ in a topological space is in the $\theta$-closure of a subset $K$ if and only if there is a net $x_{\alpha}$ in $K$ which $\theta$-converges to $x\left(x_{\alpha} \vec{\theta} x\right)$.

THEOREM 2. In any topological space

(a) the empty set and the whole space are $\theta$-closed,

(b) arbitrary intersections and finite unions of $\theta$-closed sets are $\theta$-closed,

(c) $\operatorname{cl}(K) \subset \theta-\mathrm{cl}(K)$ for each subset $K$,

(d) a $\theta$-closed subset is closed.

Received by the editors June 30, 1975.

AMS (MOS) subject classifications (1970). Primary 54D20.

Key words and phrases. $H$-closed spaces, $\theta$-closed subsets, projections. 
EXAMPLE 1. Each nonempty countable subset of the set of reals endowed with the cocountable topology is closed but not $\theta$-closed.

Main results. There are several characterizations of $H$-closed spaces in the literature [2, p. 145], [1, p. 97]. For a definition, we use the following:

Definition 4. A Hausdorff space $X$ is $H$-closed if every open cover ( $A^{\prime}$ of $X$ contains a finite subcollection 9 such that $\{\operatorname{cl}(V): V \in$.

We also make use of the following theorem immediately gotten from [11, Theorem 2]:

THEOREM 3. A Hausdorff space is $H$-closed if and only if each net in the space has a $\theta$-convergent subnet.

Definition 5. A function $g: X \rightarrow Y$ is weakly continuous [6, Theorem 6] if for each net $x_{\alpha}$ in $X$ such that $x_{\alpha} \rightarrow x$, the net $g\left(x_{\alpha}\right) \underset{\theta}{\rightarrow} g(x)$.

Definition 6. A function $g: X \rightarrow Y$ has a strongly-closed graph [6, p. 473] if for each $(x, y) \in(X \times Y)-G(g)$, there are open sets $U$ and $V$ about $x$ and $y$, respectively, such that $(U \times \operatorname{cl}(V)) \cap G(g)=\varnothing$.

It is known that a function $g: X \rightarrow Y$ has a closed graph if and only if whenever a net $x_{\alpha} \rightarrow x$ in $X$ and $g\left(x_{\alpha}\right) \rightarrow y$ in $Y$, it follows that $g(x)=y[13$, p. 115]. We have the following similar result for functions with stronglyclosed graphs.

TheOREM 4. A function $g: X \rightarrow Y$ has a strongly-closed graph if and only if whenever a net $x_{\alpha} \rightarrow x$ in $X$ and $g\left(x_{\alpha}\right) \underset{\theta}{\rightarrow} y$ in $Y$, it follows that $g(x)=y$.

Proof. Let $g$ have a strongly-closed graph and let $x_{\alpha}$ be a net in $X$ satisfying $x_{\alpha} \rightarrow x$ and $g\left(x_{\alpha}\right) \underset{\theta}{\rightarrow}$. Then $(V \times \operatorname{cl}(W)) \cap G(g) \neq \varnothing$ for $V, W$ open about $x$ and $y$ respectively. So, $(x, y) \in G(g)$ and $g(x)=y$. For the converse, let $(x, y) \in(X \times Y)-G(g)$. Then $y \neq g(x)$, and there is no net $x_{\alpha}$ in $X$ satisfying $x_{\alpha} \rightarrow x$ and $g\left(x_{\alpha}\right) \underset{\theta}{\rightarrow} y$. If $\left(V_{\sigma} \times \operatorname{cl}\left(W_{\xi}\right)\right) \cap G(g) \neq \varnothing$ for each pair $V_{\sigma}, W_{\xi}$ of sets open about $x$ and $y$ respectively, choose $\left(x_{\sigma, \xi}\right.$, $\left.g\left(x_{\sigma, \xi}\right)\right) \in\left(V_{\sigma} \times \operatorname{cl}\left(W_{\xi}\right)\right) \cap G(g)$. The ordering of $\left\{V_{\sigma} \times \operatorname{cl}\left(W_{\xi}\right): V_{\sigma}, W_{\xi}\right.$ open about $x$ and $y$ respectively $\}$ by inclusion renders $\left(x_{\sigma, \xi}, g\left(x_{\sigma, \xi}\right)\right.$ a net with $x_{\sigma, \xi} \rightarrow x$ and $g\left(x_{\sigma, \xi}\right) \underset{\theta}{\rightarrow} y$, a contradiction. Therefore, there are sets $V, W$ open about $x, y$, respectively, and satisfying $(V \times \operatorname{cl}(W)) \cap G(g)=\varnothing$; and $G(g)$ is strongly-closed. This completes the proof.

We may use the characterizations above to give a proof of the following theorem which is different and shorter than that given in [6]. If $\left(x_{\alpha}, D\right)$ is a net in a space $X$, we will denote $\left\{x_{\alpha}: \alpha>\mu\right\}$ by $T_{\mu}$ for each $\mu \in D$. Using this notation it is clear that $x_{\alpha} \theta$-converges ( $\theta$-accumulates) to a point $x \in X$ if for each open $V$ about $x$, there is a $\mu \in D$ satisfying (each $\mu \in D$ satisfies) $T_{\mu} \subset \operatorname{cl}(V)\left(T_{\mu} \cap \operatorname{cl}(V) \neq \varnothing\right)$. Let $\delta$ denote a class of topological spaces containing the class of Hausdorff completely normal and fully normal spaces.

THEOREM 5. A Hausdorff space $Y$ is $H$-closed if and only if for every space in class $S$, each $g: X \rightarrow Y$ with a strongly-closed graph is weakly continuous.

Proof. Let $Y$ be $H$-closed, let $X$ be any space and let $g: X \rightarrow Y$ have a strongly-closed graph. Let $x_{\alpha} \rightarrow x$ in $X$. Then $g\left(x_{\alpha}\right)$ is a net in $Y$, so there is a 
subnet $y_{\beta}$ of $x_{\alpha}$ and $y \in Y$ with $g\left(y_{\beta}\right) \underset{\theta}{y}$. By Theorem $4, g(x)=y$. Let $V$ be a regular open set about $g(x)$. If $g\left(x_{\alpha}\right)$ is not eventually in $\operatorname{cl}(V)$, there is a subnet $z_{\mu}$ of $x_{\alpha}$ such that $g\left(z_{\mu}\right) \theta$-converges to some $z \in Y-V$ since $Y-V$ is a regular closed set and thus $H$-closed. This then forces $g(x) \in Y-V$, a contradiction. So $g\left(x_{\alpha}\right) \underset{\theta}{\rightarrow} g(x)$. For the converse, let $x_{0} \in Y$ and let $\left(x_{\alpha}, D\right)$ be a net in $Y-\left\{x_{0}\right\}$ with no $\theta$-accumulation point in $Y-\left\{x_{0}\right\}$. Let $X=\left\{x_{\alpha}: \alpha \in D\right\} \cup\left\{x_{0}\right\}$ with the topology generated by $\left\{\left\{x_{\alpha}\right\}: \alpha \in D\right\}$ and $\left\{T_{\mu} \cup\left\{x_{0}\right\}: \mu \in D\right\}$ as the basic open sets. $X$ is a Hausdorff door space [7, p. 76] and is easily shown to be in class $S$. Let $i: X \rightarrow Y$ be the identity function and let $(x, y) \in(X \times Y)-G(i)$. If $x \neq x_{0}$, then $\{x\}$ is open in $X$; choose $V$ open in $Y$ about $y$ with $x \notin \operatorname{cl}(V)$. Then, clearly, $(\{x\} \times \operatorname{cl}(V)) \cap$ $G(i)=\varnothing$. If $x=x_{0}$, then $y \neq x_{0}$; so there is a $\mu \in D$ and a $V$ open in $Y$ about $y$ satisfying $x_{0} \notin \operatorname{cl}(V)$ and $T_{\mu} \cap \operatorname{cl}(V)=\varnothing$. So $X-\operatorname{cl}(V)$ is open in $X$ about $x$ and $[(X-\operatorname{cl}(V)) \times \operatorname{cl}(V)] \cap G(i)=\varnothing$. Thus, $i$ has a stronglyclosed graph and is weakly continuous. Consequently, if $V$ is open about $x_{0}$, there is a $\mu \in D$ satisfying $T_{\mu} \subset \operatorname{cl}(V)\left[8\right.$, p. 44], so $x_{\alpha} \underset{\theta}{\rightarrow} x_{0}$. This completes the proof.

In [6, p. 474], an example is given to show that the strongly-closed graph condition in Theorem 5 cannot be relaxed to a closed graph condition. This example was extracted from [12] and is not described explicitly in [6] presumably because of its somewhat complicated description. We now exhibit a space with a simpler description which meets the purposes of the example in [6].

Example 2. Let $N$ be the set of positive integers and let $X=\{0\} \cup[1, \infty)$ with the topology generated by the usual subspace topology of the reals on $[1, \infty)$ and $\left\{\{0\} \cup \cup_{k=m}^{\infty}(k, k+1): m \in N\right\}$ as basic open sets.

(a) The space $X$ is Hausdorff.

(b) The space $X$ is not compact since $N$ is an infinite subset of $X$ without accumulation points.

(c) The space $X$ is $H$-closed.

(d) The function $g$, from $\{1+1 / n: n \in N\} \cup\{1\}$ with the subspace topology, defined by $g(1)=1$ and $g(1+1 / n)=n$ for each $n \in N$ has a closed graph which is not strongly-closed. Also, $g$ is not weakly continuous at 1.

In [3], [4], [5], and [10], theorems of the following form are proved; $X$ has property $\lambda$ if and only if the projection $\pi_{y}: X \times Y \rightarrow Y$ is closed for each space $Y$ in a certain class. The next four theorems and main results give an analogue of this form for $H$-closed spaces.

THeOREM 6. If $X$ is an $H$-closed space then the projection from $X \times Y$ onto $Y$ takes $\theta$-closed subsets onto $\theta$-closed subsets for any space $Y$.

Proof. Let $X$ be $H$-closed, let $Y$ be any space and let $K \subset \cdot X \times Y$ be $\theta$-closed. Let $y \in \theta-\operatorname{cl}\left(\pi_{y}(K)\right)$. There is a net $\left(x_{\alpha}, y_{\alpha}\right) \in K$ with $y_{\alpha} \rightarrow \vec{\theta} y$. There is a subnet $x_{\alpha_{\mu}}$ of $x_{\alpha}$ and $x \in X$ with $x_{\alpha_{\mu}} \underset{\theta}{ } x$. So $\left(x_{\alpha_{\mu}}, y_{\alpha_{\mu}}\right) \underset{\theta}{\rightarrow}(x, y)$ and $(x$, $y) \in \theta-\operatorname{cl}(K) \subset K$. Thus $y \in \pi_{y}(K)$.

THEOREM 7. If $X$ is a Hausdorff space and the projection from $X \times Y$ onto $Y$ 
takes $\theta$-closed subsets onto closed subsets for every space $Y$, then $X$ is $H$-closed.

Proof. Let $\left(y_{\alpha}, D\right)$ be a net in $X$ with no $\theta$-convergent subnet and let $y_{0} \notin X$. Let $Y=\left\{y_{\alpha}: \alpha \in D\right\} \cup\left\{y_{0}\right\}$ with the topology generated by $\left\{\left\{y_{\alpha}\right\}\right.$ : $\alpha \in D\}$ and $\left\{T_{\mu} \cup\left\{y_{0}\right\}: \mu \in D\right\}$ as basic open sets. Let $K=\left\{\left(y_{\alpha}, y_{\alpha}\right)\right.$ : $\alpha \in D\}$ and let $(a, b) \in(X \times Y)-K$. Then $a \neq y_{0}$ and $a \neq b$. Let $V$ be open about $a$ satisfying $\left\{b, y_{0}\right\} \subset Y-\operatorname{cl}(V)$ and $T_{\mu} \subset Y-\operatorname{cl}(V)$ for some $\mu \in D$. Then $Y-\operatorname{cl}(V)$ is open and closed in $Y$ and so $V \times(Y-\operatorname{cl}(V))$ is open about $(a, b)$. Also,

$$
\operatorname{cl}[V \times(Y-\operatorname{cl}(V))] \cap K=(\operatorname{cl}(V) \times(Y-\operatorname{cl}(V))) \cap K=\varnothing .
$$

Thus, $(a, b) \notin \theta-\operatorname{cl}(K)$ and $K$ is $\theta$-closed. $\pi_{y}(K)$ is therefore closed in $Y$ and $y_{0} \in \operatorname{cl}\left(\pi_{y}(K)\right)$. This is a contradiction establishing the result.

Combining Theorems 6 and 7, we get the promised result.

TheOREm 8. A Hausdorff space $X$ is $H$-closed if and only if for every space $Y$, the projection from $X \times Y$ onto $Y$ takes $\theta$-closed subsets onto $\theta$-closed subsets.

Noting that the space $Y$ used in the proof of Theorem 7 is a Hausdorff door space and is in the class $S$ whose description precedes Theorem 5 , we may state the following theorem.

THEOREM 9. A Hausdorff space $X$ is $H$-closed if and only if for every Hausdorff door space (space in class \&) $Y$, the projection from $X \times Y$ onto $Y$ takes $\theta$-closed subsets onto $\theta$-closed subsets.

\section{REFERENCES}

1. M. P. Berri, J. R. Porter and R. M. Stephenson, Jr., A survey of minimal topological spaces, General Topology and its Relations to Modern Analysis and Algebra, III (Proc. Conf., Kanpur, 1968), Academia, Prague, 1971, pp. 93-114. MR 43 \#3985.

2. N. Bourbaki, Elements of mathematics.General topology. Part 1, Hermann, Paris; AddisonWesley, Reading, Mass., 1966. MR 34 \#5044a.

3. I. Fleischer and S. P. Franklin, On compactness and projections. Contributions to extension theory of topological structures, (Proc. Sympos., Berlin, 1967), Berlin, 1969, pp. 77-79.

4. S. Hanai, Inverse images of closed mappings. I, Proc. Japan Acad. 37 (1961), 298-301. MR $25 \# 2576$.

5. _ Inverse images of closed mappings. II, Proc. Japan Acad. 37 (1961), 302-304. MR $25 \# 2577$.

6. L. L. Herrington and P. E. Long, Characterizations of $H$-closed spaces, Proc. Amer. Math. Soc. 48 (1975), 469-475.

7. J. L. Kelley, Gernal topology, Van Nostrand, Princeton, N.J., 1955. MR 16, 1136.

8. Norman Levine, $A$ decomposition of continuity in topological spaces, Amer. Math. Monthly 68 (1961), 44-46. MR 23 \# A3548.

9. S. Mrówka, Compactness and product spaces, Colloq. Math. 7 (1959), 19-22. MR 22 \#479.

10. C. T. Scarborough, Closed graphs and closed projections, Proc. Amer. Math. Soc. 20 (1969), 465-470. MR 40 \#3514.

11. H. V. Velicko, H-closed topological spaces, Mat. Sb. 70 (112) (1966), 98-112; English transl., Amer. Math. Soc. Transl. (2) 78 (1969), 103-118. MR 33 \#6576.

12. G. Viglino, C-compact spaces, Duke Math. J. 36 (1969), 761-764. MR 40 \#2000.

13. A. Wilansky, Topology for analysis, Ginn, Waltham, Mass., 1970.

Department of Mathematics, Federal City College, Washington, D. C. 20001 